\title{
Internetassoziierte Gesundheitsängste in der hausärztlichen Versorgung - Ergebnisse einer Befragung unter Allgemein- medizinern und hausärztlich tätigen Internisten in Hessen
}

\section{Internet-Associated Health Anxieties in Primary Care - Results of a Survey among General Practitioners and Primary Care Internists in Hesse}

Autoren

Julian Wangler, Michael Jansky

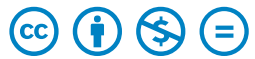

Institut

Zentrum für Allgemeinmedizin und Geriatrie, Universitätsmedizin Mainz

Schlüsselwörter

Cyberchondrie, Arzt-Patient-Verhältnis, Gesundheitsinformation, Gesundheitsängste, Hausärztliche Versorgung

Key words

cyberchondria, doctor-patient relationship, health information, health anxieties, primary care

Bibliografie

DOI https://doi.org/10.1055/a-0842-8285

Online-Publikation: 1.3.2019

Dtsch Med Wochenschr 2019; 144: e102-e108

(c) Georg Thieme Verlag KG, Stuttgart · New York ISSN 0012-0472

Korrespondenzadresse

Dr. phil. Julian Wangler

Wissenschaftlicher Mitarbeiter|Zentrum für

Allgemeinmedizin und Geriatrie

Universitätsmedizin Mainz, Am Pulverturm 13, 55131 Mainz

julian.wangler@unimedizin-mainz.de

\section{ZUSAMMENFASSUNG}

Hintergrund Gelegentlich kann es vorkommen, dass ausgedehnte Internetrecherchen bei Patienten Gesundheitsängste auslösen, die sich langfristig verfestigen. Der Umgang mit einer solchen „Cyberchondrie“ stellt eine besondere Herausforderung für den Arzt dar. Die Studie beleuchtet hausärztliche Einstellungen und Erfahrungen mit Blick auf Patienten, die vermehrt im Internet nach Symptomen, Krankheitsverläufen oder Therapien suchen. Ein Schwerpunkt gilt Personen, die aufgrund von vorangegangenen Internetkonsultationen Gesundheitsängste entwickeln. Es wird eruiert, welche Vorgehensweisen sinnvoll erscheinen, um angemessen auf verunsicherte Patienten zu reagieren.
Methoden Im Rahmen einer schriftlichen Befragung wurden zwischen dem 20. April und 20. Juni 2018 insgesamt 844 Allgemeinmediziner und hausärztlich tätige Internisten in Südhessen und zusätzlich in den Landkreisen Gießen, MarburgBiedenkopf, Kassel und der kreisfreien Stadt Kassel befragt. Ergebnisse Zwei Drittel der Befragten gehen davon aus, dass $15 \%$ oder mehr der eigenen Patienten sie mit Ergebnissen eigener Internetrecherchen konfrontieren. $73 \%$ sehen das Aufkommen von internetassoziierten Gesundheitsängsten als zunehmendes Problem in der Patientenversorgung. Es werden vor allem negative Auswirkungen der Online-Selbstinformation gesehen (psychische Stabilität, Erwartungen gegenüber dem Arzt). Jeder 5 . Arzt (18\%) hat bereits den Abbruch eines Betreuungsverhältnisses aufgrund ausgeuferter Internetrecherchen des Patienten erlebt. Um auf verunsicherte oder verängstigte Patienten zu reagieren, bauen die Befragten auf eine ausführliche Erläuterung der Diagnose und/oder Therapie und empfehlen Internetseiten, die sie als seriös erachten.

Schlussfolgerung Es erscheint ratsam, die Online-Informationssuche aktiv im Patientengespräch zu thematisieren, um möglichen negativen Auswirkungen auf das Arzt-PatientVerhältnis vorzubeugen. Entsprechend wäre darüber nachzudenken, die Anamnese um die Dimension der Online-Informationssuche zu erweitern.

\section{ABSTRACT}

Background It may happen that extensive internet search leads to health anxiety in patients, which solidify in the long term. Dealing with such 'cyberchondria' presents a special challenge for the physician. The study highlights general medical attitudes and experiences with regard to patients who are increasingly searching the Internet for symptoms, illnesses or therapies. Particular emphasis will be given to persons who develop health anxieties due to previous Health information on the Internet. It will be determined which procedures make sense in order to respond appropriately to patients with internet-related health concerns.

Methods and participants In the course of a written survey, a total of 844 general practitioners and primary care internists 
in southern Hesse and additionally in the rural districts of Gießen, Marburg-Biedenkopf, Kassel and the city of Kassel were interviewed between April 20 and June 20, 2018.

Results Two-thirds of respondents assume that $15 \%$ or more of their own patients confront them with the results of their own internet search. $73 \%$ see the emergence of internetrelated health anxiety as an increasing problem in patient care. Against this background, the respondents predominantly see negative effects caused by online self-information (psychological stability, expectations towards the doctor). Almost one of five doctors (18\%) has already experienced the termi- nation of care relationships due to an extensive internet search by the patient. In order to respond to unsettled or frightened patients, the physicians surveyed rely on a detailed explanation of the diagnosis and/or therapy and recommend certain websites that they consider to be reputable.

Discussion The authors argue that the online information search should be actively discussed in the patient interview in order to prevent possible negative effects on the doctorpatient relationship. Accordingly, it would be worth considering to extend the medical history by the dimension of online self-information.

\section{Hintergrund}

\section{Wirkungspotenziale gesundheitsbezogener Online-Recherchen}

Nationale wie internationale Studien belegen, dass inzwischen viele Patienten das Internet nutzen, um Informationen über Gesundheits- und Krankheitsthemen einzuholen [1-4]. Das Spektrum reicht von Suchmaschinen über große Gesundheitsportale oder spezielle Foren, in denen man mit Laien oder Experten in Kontakt treten kann [5-7]. Obwohl die Online-Recherche hilfreich sein kann, indem sie etwa die Einsicht in eine ärztliche Diagnose und/oder Therapie erhöht [8, 9], kann eine umfassende Suche im Internet auch Verstärker bzw. Auslöser von sich verfestigenden Gesundheitsängsten sein. Ein Beispiel hierfür sind etwa Divergenz zwischen Aussagen oder Therapievorschlägen des Arztes und Empfehlungen im Internet, die zu einem Vertrauensund Orientierungsverlust von Patienten führen können [10, 11].

Als Extremfall einer Negativwirkung von internetbasierten Gesundheitsinformationen wird das Phänomen der „Cyberchondrie“ angeführt. Angelehnt an die Hypochondrie wird unter Cyberchondrie eine durch Internetrecherchen ausgelöste extreme Angststörung oder extreme Aufmerksamkeit in Bezug auf den eigenen Gesundheitszustand verstanden [12, 13].

Die wissenschaftliche Beschäftigung mit internetassoziierten Gesundheitsängsten ist noch vergleichsweise jung; es wurden bislang nur wenige Studien vorgelegt. White und Horvitz [14] vertreten die Auffassung, dass die Internetrecherche von Patienten als quasi-diagnostische Methode eingesetzt wird, bei der mithilfe von Faktoren wie Reihenfolge, Prominenz und Informationsgehalt der Ergebnisse Rückschlüsse gezogen werden. So könnten selbst verbreitete und unspezifische Symptome als ernsthafte Erkrankung fehlinterpretiert werden.

Internationale Untersuchungen lassen einen Zusammenhang zwischen der Intensität des Online-Suchverhaltens und der Inanspruchnahme von ärztlichen Terminen, diagnostischen Verfahren sowie Gesundheitsleistungen vermuten [14-16]. Allerdings deuten Studien jüngeren Datums auch darauf hin, dass eine stark ausgeprägte Konsultation von Gesundheitsinformationen im Internet unter Umständen in eine Reduktion oder gar einen Abbruch von Arztkontakten münden kann [6].
Für den deutschsprachigen Raum wurde 2013 eine erste Studie zum Zusammenhang zwischen gesundheitsbezogener Internetrecherche und Hypochondrie vorgelegt [17]. Unter den insgesamt 471 Befragungsteilnehmern aus über 180 Online-Foren werden rund $15 \%$ als hypochondrisch klassifiziert. Diese gesundheitsängstliche Gruppe unterscheidet sich dadurch, dass sie häufiger im Internet nach eigenen Symptomen und Beschwerden sucht, die Qualität von Online-Gesundheitsangeboten merklich höher einschätzt und eine höhere Affinität zur Selbstmedikation zeigt.

\section{Sichtweise und Umgang der Ärzte}

Immer wieder wird das Phänomen beschrieben, dass in Arztpraxen zunehmend Patienten in Erscheinung treten, die aufgrund von online recherchierten Gesundheitsinformationen stark verunsichert und infolgedessen von Zweifeln, Sorgen und Ängsten bestimmt sind [18-20]. Allerdings fehlt es an Studien, die beleuchten, welche Erfahrungen Mediziner mit internetassoziierten Gesundheitsängsten gemacht haben und wie sie auf dieses durch die Internetsuche zweifelnde oder nervöse Patientenklientel reagieren.

Vorliegende Befunde beschränken sich im Wesentlichen auf die Ermittlung grundsätzlicher ärztlicher Einstellungen, wenn es im weiteren Sinne um sich selbst informierende Patienten geht [21]. So erbrachte eine Befragung von rund 800 Medizinern verschiedener Fachrichtungen, dass $78 \%$ die Auswirkungen der Selbstinformation im Versorgungsalltag häufig oder gelegentlich als kontraproduktiv und belastend empfinden [22]. Etwa ein Drittel der Ärzte hält es für richtig, Patienten, die mit selbst zusammengetragenen Informationen in die Praxis kommen, stärker in Behandlungsentscheidungen einzubeziehen, während ein weiteres Drittel dafür plädiert, sich um ausführlichere Information selbiger Patienten zu bemühen.

Eine große US-amerikanische Studie von Murray et al. [23] erbrachte, dass mit vorinformierten Patienten konfrontierte Ärzte häufig das Gefühl haben, in ihren Handlungsmöglichkeiten beschnitten zu werden. Infolgedessen wird eine Beeinträchtigung der Arzt-Patient-Beziehung erlebt und befürchtet, nicht mehr effektiv auf den Patienten einwirken zu können [24].

Die hausärztliche Versorgung ist in besonderer Weise von internetassoziierten Gesundheitsängsten tangiert. Zum einen werden Allgemeinmediziner mit der vollen Bandbreite an 
Gesundheits- und Krankheitsthemen konfrontiert und haben deshalb auch mit einer sehr heterogenen Klientel gesundheitsängstlicher Patienten zu tun. Zum anderen obliegt es Hausärzten, auf verunsicherte Patienten so einzuwirken, dass diese stabilisiert werden und ggf. einer angemessenen Weiterversorgung zugänglich gemacht werden können.

\section{Methode}

\section{Erkenntnisinteresse und Fragebogen}

Um einen Beitrag zur Erweiterung des noch stark lückenhaften Forschungsstandes zu leisten und die ärztliche Perspektive hierbei stärker zu berücksichtigen, hat die Abteilung Allgemeinmedizin der Universitätsmedizin Mainz zwischen dem 20. April und 20. Juni 2018 eine schriftliche Befragung unter Hausärzten und hausärztlich tätigen Internisten durchgeführt. Folgende Fragen standen im Zentrum der explorativ angelegten Untersuchung:

- Welche Ansichten vertreten hausärztlich tätige Mediziner in Bezug auf Patienten, die sich regelmäßig im Internet über Gesundheit und Krankheit informieren? Welche Auswirkungen hat die Recherche nach Ansicht bzw. Erfahrung der Ärzte?

- Wie häufig haben hausärztlich tätige Mediziner im Praxisalltag mit Patienten zu tun, die aufgrund von vorangegangenen Internetrecherchen Ängste entwickeln?

- Welche Vorgehensweisen werden für sinnvoll befunden, um angemessen auf entsprechend verunsicherte Patienten zu reagieren?

Der Fragebogen wurde zum einen auf Grundlage einer Literaturrecherche erstellt. Hier fanden die erwähnten Patienten- und Ärztebefragungen Berücksichtigung. Zum anderen wurden Vorgespräche mit insgesamt sieben Hausärzten geführt, die für die Konkretisierung und Fokussierung des Fragebogens entscheidend waren und im Speziellen der Generierung einer Itembatterie (Frage 7) dienten. Vor dem Feldeinsatz wurde ein Pretest durchgeführt.

Der Fragebogen (Ausfülldauer ca. 10 Minuten) setzt sich aus drei inhaltlichen Schwerpunkten zusammen: Einstellungen im Hinblick auf online recherchierende Patienten (Fragen 1 und 2, teilweise Itembatterie in Frage 7), (Verhaltens-) Beobachtungen und Charakteristika online recherchierender Patienten (Fragen 3 bis $5,8,11$ ), ärztliche Vorgehensweisen gegenüber online recherchierenden bzw. aufgrund der Online-Recherche verunsicherten Patienten (Fragen 6, 9, 10, teilweise Itembatterie in Frage 7).

\section{Ärzterekrutierung}

Auf schriftlichem Weg zur Teilnahme an der anonymisierten Befragung eingeladen wurden sämtliche als Behandler aktive Allgemeinmediziner und hausärztliche Internisten in Südhessen (Regierungsbezirk Darmstadt). Hinzu kamen in Nordhessen die Landkreise Gießen, Marburg-Biedenkopf, Kassel und die kreisfreie Stadt Kassel. Insgesamt angeschrieben wurden ca. 3300 Ärzte, davon rund 2500 Hausärzte und rund 800 hausärztlich tätige Internisten.

\section{Soziodemografie und Datenanalyse}

Als soziodemografische Merkmale wurden Alter, Geschlecht, Facharzthintergrund, Praxisumgebung, Praxisform und Patienten pro Quartal erhoben. Zudem wurde die Einwohnerzahl des Praxisorts grob klassiert ermittelt sowie nach dem Vorhandensein einer Weiterbildung im Bereich Psychotherapie oder Psychoanalyse gefragt.

Nach Bereinigung des Datensatzes wurden die Daten mittels SPSS 23.0 für Windows ausgewertet.

\section{Ergebnisse}

\section{Stichprobe}

Von den insgesamt 865 zurückgeschickten Fragebögen gingen die 844 vollständig ausgefüllten Bögen in die Auswertung ein. Gemessen an der Gesamtzahl aller angeschriebenen Ärzte beträgt die Rücklaufquote rund $26 \%$. Die Stichprobe lässt sich wie folgt beschreiben:

- Geschlecht: $54 \%$ männlich, $46 \%$ weiblich

- Facharzt: $77 \%$ Allgemeinmediziner, $22 \%$ Internisten (hausärztlich tätig), 1 \% Sonstiges

- Praxisumgebung: 46\% mittel- und großstädtisch, 54\% ländlich-kleinstädtisch

- Praxisform: 49\% Einzelpraxen, 46\% Gemeinschaftspraxen, $5 \%$ Sonstiges

- Durchschnittsalter: 55 Jahre

- Weiterbildung Psychotherapie oder Psychoanalyse: $8 \%$

\section{Einstellungen und Erfahrungen bezüglich online recherchierender Patienten}

Die Möglichkeiten im Internet, sich über Gesundheits- und Krankheitsthemen zu informieren, werden vom größten Teil des Samples eher kritisch eingeschätzt. So sind $47 \%$ der Auffassung, die Inanspruchnahme des Internets habe eher negative Folgen für das Arzt-Patient-Verhältnis (eher positive Folgen: $18 \%$, unentschieden: $35 \%$ ). Etwas besser werden die Folgen für das Gesundheitsverhalten von online recherchierenden Patienten eingeschätzt (eher positive Folgen: $26 \%$, eher negative Folgen: $43 \%$, unentschieden: $31 \%$ ). Hierbei bestehen keinerlei Unterschiede zwischen älteren und jüngeren Medizinern oder zwischen städtischen und ländlichen Praxisumgebungen.

Fragt man nach den konkreten Effekten für das Arzt-PatientVerhältnis, wenn Patienten sich vermehrt gesundheitsbezogene Informationen aus dem Internet beschaffen, so beobachtet eine Mehrheit der befragten Mediziner ein beträchtliches Verwirrungsund Verunsicherungspotenzial von Online-Recherchen bei ihren Patienten ( $\vee$ Tab. 1). Dieses kann in Nervosität und Gesundheitsangst umschlagen oder etwa aufgrund von falschen oder widersprüchlichen Angaben inkorrekte Erwartungen auf Patientenseite, eine Verschlechterung der Compliance oder gar eine steigende Bereitschaft zur Selbstmedikation nach sich ziehen. Nur ein kleinerer Teil der Befragten glaubt, dass die regelmäßige Suche im Internet zu einer Verbesserung der Informiertheit und tieferer Einsicht in ärztliche Diagnosen und Handlungsweisen führen können. 
- Tab. 1 Das Verhältnis zwischen Arzt und Patient kann sich verändern, wenn Patienten sich vermehrt oder sogar regelmäßig Informationen zu Gesundheit und Krankheit aus dem Internet beschaffen. (Mehrfachauswahl war möglich; $N=844$ ).

\section{Welche der folgenden Punkte treffen Ihrer Ansicht} bzw. Erfahrung nach zu?

Patienten sind von Informationen aus dem Internet verwirrt und verunsichert.

Patienten stellen mehr Fragen.

Patienten werden nervöser, ängstlicher.

Patienten sind kritischer gegenüber dem Arzt.

Patienten kommen mit falschen Erwartungen in die Sprechstunde.

Patienten bereiten Arztbesuch mit Internetrecherchen nach, um z. B. Diagnose zu prüfen.

Patienten tendieren zur Selbstmedikation.

Die Compliance der Patienten leidet unter Internetrecherchen.

Patienten kommen häufiger in die Sprechstunde.

Patienten haben weniger Vertrauen zum Arzt.

Patienten sind konfliktbereiter.

Patienten sind besser informiert, können den Arzt besser verstehen.

Patienten kommen bei Beschwerden rechtzeitiger.

Patienten gehen aufgrund häufiger

Internetrecherchen nicht rechtzeitig zum Arzt.

Patienten meiden oder umgehen Arztbesuche häufiger.

Patienten fühlen sich sicherer.

$6 \%$

Patienten agieren durch regelmäßige

Internetrecherchen vernünftiger.

Internetrecherchen haben keinen Einfluss auf

Patienten.

Ärzte, die grundsätzlich positive Auswirkungen von Internetrecherchen auf das Gesundheitsverhalten von Patienten sehen, betonen im Vergleich zu negativ eingestellten Befragten besonders stark, dass Patienten den Arzt aufgrund der eingeholten Informationen besser verstehen können (56 zu 13\%) und bei Beschwerden rechtzeitiger vorstellig werden (32 zu 9\%).

\section{(Verhaltens-) Beobachtungen und Charakteristika online recherchierender Patienten}

Wie häufig Ärzte von ihren Patienten auf selbst recherchierte gesundheitsbezogene Informationen aus dem Internet angesprochen werden, hat eine Folgefrage ermittelt. Hierbei schätzen $66 \%$ der Ärzte, dass $15 \%$ oder mehr der eigenen Patientenschaft sie schon mal oder häufiger mit den Ergebnissen eigener Internetsuchen konfrontiert (bis zu 10\%: 24\%). Üblicherweise beziehen sich die Informationen, die die entsprechenden Patienten im Internet konsultieren, auf bestimmte Krankheitsbilder (91\%) bzw. -symptome (89\%), Therapien (66\%), Diagnostik (55\%) und neue Medikamente (48\%). Andere Aspekte (z. B. Prävention, gesunde Lebensweise, ärztliche Zusatzleistungen) spielen bei den Recherchen eine untergeordnete Rolle.

Des Weiteren wurde nach typischen Merkmalen von Patienten gefragt, die den Arztbesuch regelmäßig mit Internetsuchen vorund nachbereiten. Nach Angabe der befragten Mediziner handelt es sich überwiegend um Personen unter 60 Jahren (82\%). Als weitere Eigenschaften werden von $42 \%$ psychosomatische (Vor-) Erkrankungen genannt. $32 \%$ sind der Auffassung, es handele sich meist um Personen mit höherer Bildung.

Mit dem Phänomen, dass Patienten aufgrund vorangegangener Online-Recherchen stark verunsichert sein können und infolgedessen befürchten, eine schlimme Erkrankung zu haben, obwohl aus ärztlicher Sicht keine entsprechenden Hinweise bestehen, sind die Befragten vertraut. So geben drei Viertel aller Ärzte an, dass ihnen eine solche unbegründete Angst vor ernsten Krankheiten, die auf der Zurkenntnisnahme von Internetinhalten basiert, bereits häufig (21\%) oder gelegentlich (54\%) aufgefallen ist (selten: $23 \%$, nie: $2 \%$ ). $18 \%$ haben nach eigener Angabe schon einmal oder mehrmals erlebt, dass eine Patientenbetreuung von hausärztlicher oder Patientenseite beendet wurde, weil der Patient durch sein Informationsverhalten im Internet so stark beeinflusst wurde, dass eine Weiterbetreuung nicht mehr möglich war.

Wie eine weitere Itembatterie mit vorgegebenen Aussagen bestätigt, nehmen die befragten hausärztlichen Mediziner Patienten, die aufgrund von intensiven Internetrecherchen beunruhigt sind, als zunehmende Herausforderung im Versorgungsalltag wahr ( $\triangleright$ Abb. 1). Aus ihrer Erfahrung erwarten sie bei dieser Patientenklientel einen größeren Diagnostik- und Beratungsaufwand, für den jedoch nicht immer die erforderlichen Zeitressourcen vorhanden sind. In diesem Zusammenhang wird auch als Problem erlebt, dass es bis dato keine entsprechende Beratungsund Abrechnungsziffer für internetassoziierte Betreuungsleistungen gibt. Ärzte mit einer Zusatzbezeichnung Psychotherapie oder Psychoanalyse geben deutlich häufiger an, dass Hausärzte sich stärker als Ansprechpartner für internetinformierte Patienten positionieren sollten als Ärzte ohne entsprechende Weiterbildung (87 zu $61 \%$ ).

Der abschließende Komplex des Fragebogens befasst sich mit ärztlichen Umgangsstrategien und Vorgehensweisen, um internetassoziierten Gesundheitsängsten entgegenzuwirken bzw. diese zu verhindern. 38 \% der Befragten geben an, sich bei bestimmten Patientengruppen prinzipiell danach zu erkundigen, inwiefern im Vorfeld des Arztbesuchs Vorrecherchen im Internet durchgeführt wurden; 21 \% bekunden, diese Internet-Vorrecherchen im ArztPatient-Gespräch sehr stark oder eher stark zu berücksichtigen. Unterschiede zwischen psychotherapeutisch bzw. -analytisch weitergebildeten Ärzten und Ärzten ohne eine solche Weiterbildung fallen hier noch deutlicher aus. So fragen $89 \%$ der ersteren und $32 \%$ der letzteren nach Vorrecherchen im Internet.

Des Weiteren kommt es nach Angabe von $46 \%$ der Befragten häufig oder gelegentlich vor, dass sie Patienten bestimmte Internetseiten empfehlen, die nach Ansicht der Befragten als seriöse und zuverlässige Quellen für gesundheitsbezogene Informationen einzustufen sind. 
„Patienten, die sich im Internet intensiv zu ihrer Krankheit oder Symptomen informieren, fordern häufiger eine weiterführende apparative Diagnostik ein."

"Patienten, die aufgrund übertriebener
Internetrecherchen stark beunruhigt sind, sind ein
zunehmendes Problem in der ärtlichen
Versorgung."

„Die Gebührenordnung sollte vorsehen, ärtliche Leistungen zu honorieren, die im Zusammenhang mit der Beratung in Fragen der internetbasierten Gesundheitsrecherche erbracht werden."

„Hausärzte sollten stärker signalisieren, dass Sie der Ansprechpartner für Patienten sind, die sich im Internet zu Gesundheit und Krankheit informieren."

„Es fehlt im Praxisalltag häufig die nötige Zeit, um sich als Hausarzt mit von Patienten recherchierten Internet-Informationen auseinanderzusetzen bzw. Stellung dazu zu nehmen."
„Patienten, die intensiv im Internet zu Gesundheit und Krankheit recherchieren, sind in der Regel auch besser informiert und wissen besser Bescheid."

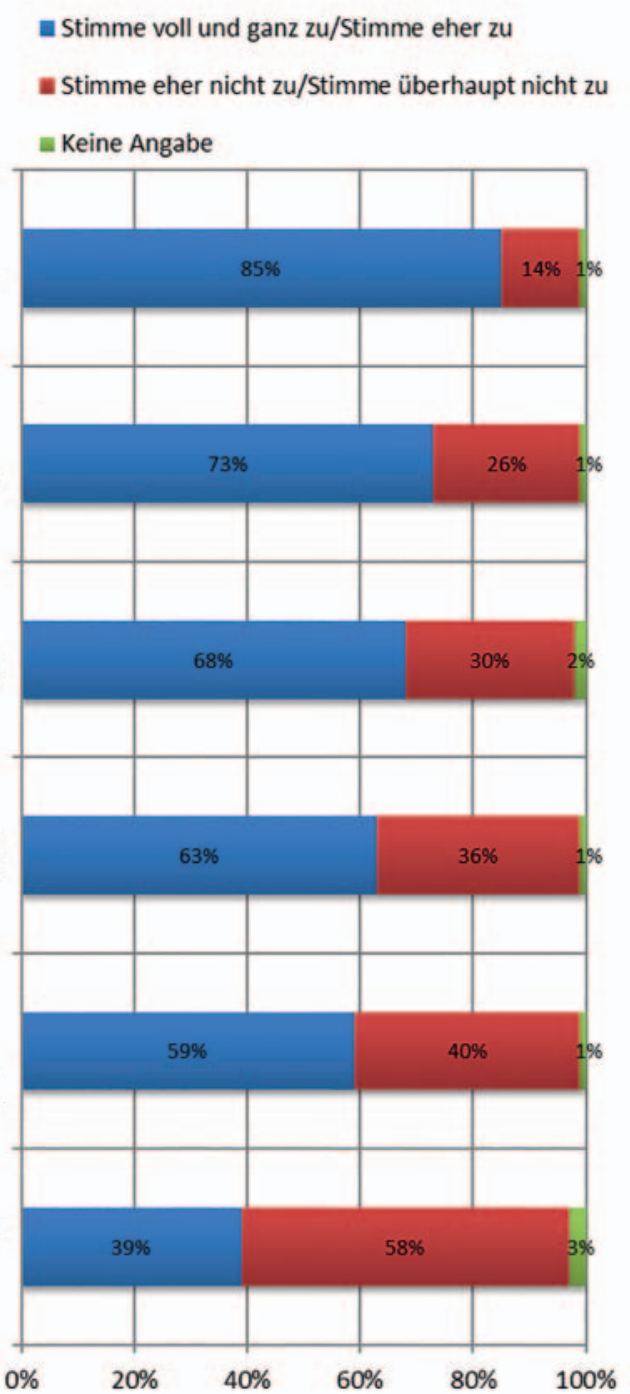

- Abb. 1 Wie stark stimmen Sie den folgenden Aussagen zu? $(N=844)$.

Fragt man hausärztlich tätige Mediziner, welche Vorgehensweisen sie für angebracht halten, um den durch Internetrecherche verunsicherten Patienten zu helfen, verlassen sich Ärzte nach wie vor in erster Linie auf eine ausführliche Erläuterung zu Diagnose und Therapie, ggf. räumen sie mehr Beratungszeit ein ( $\triangleright$ Tab. 2). Auch Hinweise auf seriöse Gesundheitsinformationen im Internet oder das Aushändigen von Broschüren mit Hintergrundinformationen werden mehrheitlich als sinnvoll erachtet. Die wenigsten Ärzte halten es hingegen für angebracht oder praktikabel, Patienten grundsätzlich von eigeninitiativer Informationssuche im Internet abzuraten.

Befragte mit einer Zusatzbezeichnung Psychotherapie bzw. Psychoanalyse geben deutlich häufiger an, sich gemeinsam mit dem Patienten mit recherchierten Informationen auseinanderzusetzen (74 zu 46\%), vom Patienten recherchierte Informationen zu überprüfen und anschließend Rücksprache zu halten (69 zu 39\%) sowie grundsätzlich Potenziale und Risiken einer Online-Recherche im Rahmen der Sprechstunde zu thematisieren (84 zu 33\%).

\section{Diskussion}

\section{Zusammenfassung}

Die Befragung von 844 Allgemeinmedizinern und hausärztlich tätigen Internisten in Hessen zeigt, dass es für Ärzte inzwischen Normalität ist, dass Patienten sich regelmäßig im Internet zu Gesundheits- und Krankheitsthemen informieren. Drei Viertel der Hausärzte beobachten allerdings als zunehmendes Problem, dass bei einem Teil ihrer Patienten aufgrund von intensiven Online-Konsultationen Gesundheitsängste aufkommen. Entsprechend negativ erleben die Befragten im Praxisalltag oftmals die Auswirkungen solcher gesundheitsbezogenen Eigenrecherchen, bei denen sie vor allem beeinträchtigende Folgewirkungen für die psychische Stabilität von Patienten, die Erwartungen gegenüber dem Arzt oder auch die Bereitschaft zur Selbstmedikation und die Compliance sehen. Immerhin fast jeder fünfte Arzt hat inzwischen persönlich den Abbruch eines oder mehrerer Betreu- 
- Tab. 2 Bei Patienten, deren psychische Gesamtsituation durch Internetrecherchen negativ beeinflusst werden könnte, kann der Hausarzt bestimmte Maßnahmen ergreifen, um insbesondere dem Aufkommen von Gesundheitsängsten entgegenzuwirken. (Mehrfachauswahl war möglich; $\mathrm{N}=844$ ).

Welche der folgenden Punkte halten Sie für erfolgversprechend und praktikabel?

ausführliche Erläuterung z. B. zu Diagnose und Therapie, um einer ausufernden oder ziellosen Internetrecherche des Patienten vorzubeugen (ggf. Einräumen von mehr Beratungszeit)

Hinweise auf seriöse Informationsquellen im Internet zu entsprechenden Themengebieten (z. B. anerkannte Gesundheitsportale) dem Patienten als Tipp mitgeben vertrauenswürdige Informationsmaterialen (z. B. Broschüren) aushändigen

gemeinsame Auseinandersetzung mit den vom Patienten recherchierten Informationen bzw. Internetseiten

Erweiterung des typischen Anamnesefragebogens um die Erfassung der Häufigkeit von Internetrecherchen zu Gesundheit und Krankheit seitens des Patienten, sodass der Arzt frühzeitig auf vorhandene oder entstehende Gesundheitsängste aufmerksam werden kann

Überprüfung der vom Patienten recherchierten Informationen und Rücksprache mit dem Patienten (z. B. zur Richtigstellung)

grundsätzliche Thematisierung von Potenzialen und Risiken einer Online-Recherche im Rahmen der Sprechstunde

prinzipiell von eigeninitiativer Informationssuche im Internet abraten

ungsverhältnisse erlebt, weil das Verhältnis zum Patienten durch sein unkontrolliertes Online-Informationsverhalten nicht mehr möglich war.

Um auf verunsicherte oder verängstigte Patienten zu reagieren bzw. einer Verunsicherung in der Zukunft vorzubeugen, ist ein Teil der Ärzte dazu übergegangen, bei bestimmten Patientengruppen vorsorglich nach durchgeführten Recherchen zu fragen und diese entsprechend im Arzt-Patient-Gespräch zu berücksichtigen. Insgesamt bauen die befragten Mediziner darauf, internetassoziierte Gesundheitsängste vor allem dadurch zu verhindern, dass sie sich ausreichend Zeit für eine Erläuterung von Diagnose und/oder Therapie nehmen und zur Nachbereitung oder weiteren Recherche bestimmte Internetseiten empfehlen, die sie als seriös erachten. Dabei fällt bei Ärzten, die über eine Zusatzbezeichnung Psychotherapie oder Psychoanalyse verfügen, eine merklich höhere Bereitschaft auf, sich mit den Online-Vorrecherchen von Patienten zu beschäftigen, darüber Rücksprache mit ihnen zu halten und auf Chancen und Gefahren einer Internetrecherche im Rahmen der Sprechstunde hinzuweisen.

\section{Stärken und Schwächen}

Aufgrund der begrenzten Fallzahl und des regionalen Rekrutierungsschwerpunktes vornehmlich in Südhessen kann die Studie keinen repräsentativen Anspruch erheben. Zudem kann nicht ausgeschlossen werden, dass thematisch interessiertere bzw. kompetentere Ärzte in stärkerem Maße an der Erhebung teilgenommen haben. Dennoch konnte aufgrund eines zufriedenstellenden Rücklaufs eine heterogene Stichprobe gewonnen werden, die einen beträchtlichen Teil der in Hessen praktizierenden Hausärzte beinhaltet und entsprechend in die Breite der Hausärzteschaft hineinreicht.

Schließlich ist darauf hinzuweisen, dass die vorliegende Befragung keine Aussagen darüber treffen kann, wie sich Gesundheitsängste bei Patienten im Zusammenhang mit intensiven Internetrecherchen dynamisch entwickeln und individuell manifestieren, d. h. inwiefern sie durch bestimmte Voraussetzungen begünstigt werden und mit anderen (intervenierenden) Faktoren interagieren. Um genaueren Aufschluss hierüber zu erhalten, wäre der Einsatz qualitativer Interviews bzw. Fokusgruppen anzuregen.

\section{Schlussfolgerung}

Inzwischen gehört es für Ärzte zum Versorgungsalltag, dass Patienten sich vor und nach dem Arztbesuch online Informationen zu Gesundheit und Krankheit beschaffen [4-8]. Dies bleibt nicht ohne Folgen für das Arzt-Patient-Verhältnis, sondern kann sowohl direkt als auch indirekt Einfluss auf das Gesundheitsverhalten von Patienten, ihr Auftreten während des Arztgesprächs oder die Compliance nehmen [1, 6, 15, 16]. In manchen Fällen kann es vorkommen, dass ausgedehnte Internetrecherchen bei Patienten Gesundheitsängste auslösen, die sich langfristig verfestigen $[17,19]$. Der ärztliche Umgang mit einer solchen „Cyberchondrie" gestaltet sich herausfordernd. Dessen sind sich viele Ärzte bereits bewusst. Insgesamt weisen die Befunde darauf hin, dass Ärzte begonnen haben, sich auf die Problematik internetassoziierter Gesundheitsängste einzulassen und nach Lösungen zur (präventiven) Stabilisierung von Patienten zu suchen.

Es erscheint sinnvoll, in der täglichen Sprechstunde aktiv auf internetbasierte Gesundheitsrecherchen einzugehen, deren Potenziale und Risiken zu thematisieren und für die Arzt-PatientBeziehung zu nutzen. Indem der Arzt auf die Recherchen des Patienten eingeht, kann er möglichen Verunsicherungen nicht nur besser vorbeugen, sondern signalisiert zugleich auch Wertschätzung. Beides kommt der Patientenbindung zugute. Vor diesem Hintergrund wäre auch darüber nachzudenken, die Anamnese um die Dimension der (Online-) Informationssuche zu erweitern. Weiter sollte berücksichtigt werden, dass gesundheitsängstliche oder durch widersprüchliche Informationen im Internet verunsicherte Patienten ggf. mehr Beratungszeit benötigen. Nicht zuletzt wäre, wie Bittner anregt, darüber nachzudenken, die Bekanntheit guter, seriöser Informationsangebote nicht lediglich bei Laien, sondern auch in Fachkreisen zu stärken [22, 25]. 


\section{KERNAUSSAGEN}

- In Arztpraxen treten zunehmend Patienten in Erscheinung, die aufgrund von online recherchierten Gesundheitsinformationen stark verunsichert und infolgedessen von Zweifeln, Sorgen und Ängsten betroffen sind.

- Drei Viertel der befragten Hausärzte sehen als zunehmendes Problem, dass bei einem Teil ihrer Patienten aufgrund von intensiven Online-Konsultationen Gesundheitsängste aufkommen. Auswirkungen solcher gesundheitsbezogenen Eigenrecherchen im Internet betreffen die psychische Stabilität von Patienten, die Erwartungen gegenüber dem Arzt oder auch die Bereitschaft zur Selbstmedikation und die Compliance.

- Knapp jeder fünfte Arzt hat bereits den Abbruch eines oder mehrerer Betreuungsverhältnisse erlebt, weil das Verhältnis zum Patienten durch sein unkontrolliertes Online-Informationsverhalten nicht mehr möglich war.

- Angesichts der untersuchten Problematik sollte darüber nachgedacht werden, die Online-Informationssuche aktiv im Patientengespräch zu thematisieren, um möglichen negativen Auswirkungen auf das Arzt-Patient-Verhältnis vorzubeugen. Entsprechend wäre darüber nachzudenken, die Anamnese um die Dimension der Online-Informationssuche zu erweitern.

Interessenkonflikt

Die Autoren geben an, dass kein Interessenkonflikt besteht.

Literatur

[1] Braun B, Marstedt G. Non-Compliance bei der Arzneimitteltherapie: Umfang, Hintergründe, Veränderungswege. In: Bocken J, Braun B, Landmann J, Hrsg Gesundheitsmonitor 2011. Gütersloh: Bertelsmann Stiftung; 2011: 56-76

[2] Taylor H. Cyberchondriacs on the Rise? The Harris Poll \#95, August 4th, 2010.

[3] Europäische Kommission. Europeans becoming enthusiastic users of online health information (28.11.2014). Im Internet: https://ec.europa.eu/ digital-single-market/en/news/europeans-becoming-enthusiastic-usersonline-health-information; Stand: 25.10.2018

[4] Eichenberg C, Brähler E. Das Internet als Ratgeber bei psychischen Problemen: Eine bevölkerungsrepräsentative Befragung in Deutschland. Psychotherapeut 2013; 58: 63-72. doi:10.1007/s00278-012-0893-0

[5] Cassidy JT, Baker JF. Orthopaedic Patient Information on the World Wide Web: An Essential Review. J Bone Joint Surg Am 2016; 98 (4): 325 - 338. doi:10.2106/JBJS.N.01189

[6] Wangler J, Jansky M. Wie wirken sich Gesundheitsportale auf das ArztPatienten-Verhältnis aus? Zeitschrift für Allgemeinmedizin 2017; 93: 278-282. doi:10.3238/zfa.2017.0278-0282

[7] Bertelsmann Stiftung: Wer suchet, der findet - Patienten mit Dr. Google zufrieden. Gesundheitsinfos Daten, Analysen, Perspektiven. Nr. 2, 2018 (Januar 2018). Im Internet: http://daebl.de/GH88; Stand: 25.10.2018

[8] Baumann E, Czerwinski F. Erst mal Doktor Google fragen? Nutzung neuer Medien zur Information und zum Austausch über Gesundheitsthemen. In:
Böcken J, Braun B, Meierjürgen R, Hrsg Gesundheitsmonitor 2015. Bürgerorientierung im Gesundheitswesen. Gütersloh: Bertelsmann Stiftung; 2015: $57-79$

[9] Baumann E, Link E. Onlinebasierte Gesundheitskommunikation: Nutzung und Austausch von Gesundheitsinformationen über das Internet. In: Fischer F, Krämer A, Hrsg eHealth in Deutschland. Anforderungen und Potenziale innovativer Versorgungsstrukturen. Berlin/Heidelberg: Springer; 2016: 385 -406. doi:10.1007/978-3-662-49504-9_21

[10] Baker L, Wagner TH, Singer $S$ et al. Use of the Internet and e-mail for health care information. J Am Med Assoc 2003; 289: 2400-2406. doi:10.1001/jama.289.18.2400

[11] Weaver ], Thompson N, Weaver S et al. Profiling characteristics of individual's using Internet health information in health care adherence decision. Paper präsentiert auf der 136. Jahrestagung der American Public Health Association, San Diego, 25. - 29. Oktober 2008

[12] YouGov. Dr. Internet: Online-Diagnose statt Arztbesuch? Patientenbefragung im Auftrag der Siemens-Betriebskrankenkasse (28.05.2015). Im Internet: https://www.sbk.org/uploads/media/pm-dr-internet-onlinediagnose-statt-arztbesuch-sbk_150528.pdf; Stand: 25.10.2018

[13] Eichenberg C, Wolters C. Cyberchondria. In: Brähler E, Hoefert HW, Hrsg Lexikon der Modernen Krankheiten - Phänomene, Gefahren, Irrtümer. Berlin: MWV; 2015: 114-118

[14] White RW, Horvitz E. Cyberchondria: Studies of the Escalation of Medical Concerns in Web Search. ACM Transactions on Information Systems (Microsoft Research Technical Report) 2009; 27: 23:1 -23:37. doi:10.1145/1629096.1629101

[15] Eastin MS, Guinsler NM. Worried and Wired: Effects of Health Anxiety on Information-Seeking and Health Care Utilization Behaviors. Cyberpsychol Behav 2006; 9: 494 - 498. doi:10.1089/cpb.2006.9.494

[16] Barke A, Blechhardt G, Rief W et al. The Cyberchondria Severity Scale (CSS): German Validation and Development of a Short Form. Int J Behav Med 2016; 23: 595-605. doi:10.1007/s12529-016-9549-8

[17] Eichenberg C, Wolters C. Cyberchondrie, ein modernes Symptom? NeuroTransmitter 2013; 7/8: 28 -32. doi:10.1007/s00278-011-0861-0

[18] Eichenberg C, Wolters C. Gesundheitsängste und das Internet: Cyberchondrie oder ,Der eingebildete Kranke 2.0'. Orthopädie \& Rheuma 2014; 17: 12 - 15. doi:10.1007/s15012-014-0020-8

[19] Eichenberg C. Gesundheitsängste und Internet. In: Hoefert W, Klotter C, Hrsg Gesundheitsängste. 2012 Lengerich: Pabst; 2012: 239-263

[20] Powell J, Inglis N, Ronnie J et al. The characteristics and motivations of online health information seekers: cross-sectional survey and qualitative interview study. J Med Internet Res 2011; 13: e20. doi:10.2196/ jmir. 1600

[21] Streich W. Der informierte Patient - Hinweise zum praktischen Nutzen seines Wissens in der ambulanten Versorgung. Gesundheitsmonitor Newsletter März 2004. Gütersloh, 2004

[22] Bittner A. Erfahrungen, Einstellungen und Umgang von Ärzten mit informierten Patienten. In: Böcken J, Braun B, Meierjürgen R, Hrsg Gesundheitsmonitor 2016. Bürgerorientierung im Gesundheitswesen. Gütersloh: Bertelsmann Stiftung; 2016: 141-159

[23] Murray E, Lo B, Pollack L et al. The Impact of Health Information on the Internet on Health Care and the Physician-Patient Relationship: National U.S. Survey among 1050 U.S. Physicians. Journal of Medical Internet Research 2003; 5 (3): e17. doi:10.2196/jmir.5.3.e17

[24] Baumgart ]. Ärzte und informierte Patienten: Ambivalentes Verhältnis. Dtsch Ärztebl 2010; 107 (51): A 2554-A2556

[25] Bittner J. Vermittlung von Gesundheitskompetenz durch für Patienten verständliche medizinische Befunde (16.12.2015). Im Internet: https:// d-nb.info/1081788747/34; Stand: 25.10.2018 